\title{
Comments on "A Method for Correcting Airborne Temperature Data for Sensor Response Time"
}

\author{
Donald T. Acheson \\ Environmental Data Service, NOAA, Washington, D.C. 20235
}

27 March 1973

The article by McCarthy (1973) has a methodology which is both risky and lacking in physical insight.

The procedure used by the author begins with an analytical expression [(3)] of experimental data describing the response of a sensor to a step-function input and derives a differential equation $[(9)]$ which is used to apply response corrections to a much more general class of input data. This strikes me as finding the problem to fit a solution.

As a simple example of the risk involved, imagine an experimenter displacing a weight on a spring, releasing it, and observing simple harmonic motion described by $Z=Z_{0} \cos \omega t$. This is the solution to at least two differential equations:

$$
\begin{aligned}
d^{2} Z / d l^{2}+\omega^{2} Z & =0 \\
d Z / d t+(\omega \tan \omega t) Z & =0 .
\end{aligned}
$$

If the experimenter ignored his physics and foolishly chose the second differential equation as the proper description of the mass-spring system, disasterous predictions of system response to more general inputs than a step function would follow.

More directly, consider the following simple model to represent the heat transfer between the sensor system described by the author and the ambient air. Ambient air at temperature $T_{a}$ flows through a cylinder at temperature $T_{c}$ and then over a thermometer at temperature $T_{i}$ as shown below:

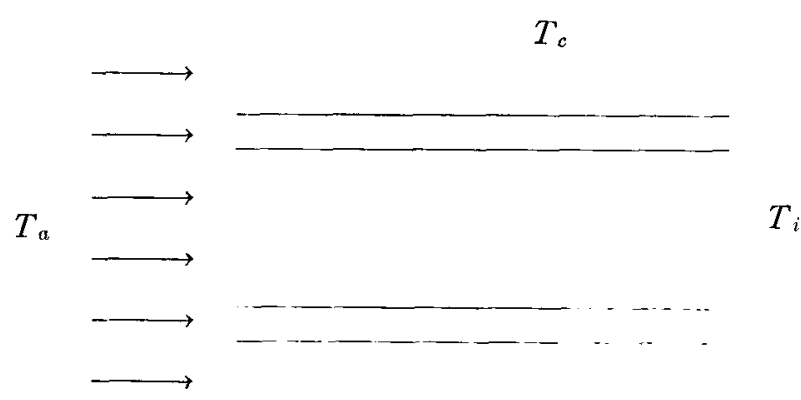


Assume $T_{c}$ and $T_{i}$ to be governed by the differential equations

$$
\begin{aligned}
& d T_{c} / d t=k_{1}\left(T_{a}-T_{c}\right), \\
& d T_{i} / d t=k_{2}\left(A T_{c}+B T_{a}-T_{i}\right),
\end{aligned}
$$

in which $A+B=1$ and the term $A T_{c}+B T_{a}$ parameterizes the heat transfer and mixing going on before the air reaches the thermometer. Using the first to eliminate $T_{c}$ from the second, one obtains

$$
\frac{d^{2} T_{i}}{d t^{2}}+\left(k_{1}+k_{2}\right) \frac{d T_{i}}{d t}+k_{1} k_{2} T_{i}=k_{1} k_{2} T_{a}+B k_{2} \frac{d T_{a}}{d t} .
$$

This last differential equation not only bears a strong resemblance to Eq. (9) in the journal paper, but also has Eq. (3) as a solution.

Either Eq. (9) or my second-order differential equation can be written in the form

$$
\bar{\lambda} \frac{d T_{a}}{d t}+T_{a}=T_{i}+\left(\lambda_{1}+\lambda_{2}\right) \frac{d T_{i}}{d t}+\lambda_{1} \lambda_{2} \frac{d^{2} T_{i}}{d t^{2}}
$$

where $\lambda_{1}=1 / k_{1}$ and $\lambda_{2}=1 / k_{2}$ for both equations, while $\bar{\lambda}=A \lambda_{2}+B \lambda_{1}$ in the author's version and $\bar{\lambda}=B \lambda_{1}$ in mine. From the form of the equation, $\bar{\lambda}$ behaves like a time constant. Of course, errors in its estimation produce errors in the solution for $T_{a}$. Using values from the author's Table 1 for $A, B, \lambda$ and $\lambda_{2}$ shows that the differences between the two values of $\bar{\lambda}$ are only a few percent. Probably no significant differences would appear in Fig. 3 in the article had the author used my expression for $\bar{\lambda}$ instead of his own. However, this is fortuitous in that $A \lambda_{2} \ll B \lambda_{1}$ for both the NCAR and Rosemount temperature sensors.

The point I wish to stress is not that my differential equation is better than the author's (which may or may not be so), but rather that the method of proceeding from solution to differential equation does not produce correct results in general. It is no substitute for physical modeling of a system.

\section{REFERENCE}

McCarthy, John, 1973: A method for correcting airborne temperature data for sensor response time. $J$. Appl. Meteor., $12,211-214$. 